\title{
Painless arm swelling in a young woman
}

\author{
F de la Iglesia Martínez, C Pellicer Vázquez, V Ramos Polledo, M Marini Díaz, \\ F López Fernández, F Diz-Lois Martínez
}

The patient was a 39-year-old woman who, after a brisk movement of the head, noticed pain in the cervical region. Twenty hours later she began to have swelling of the left superior limb. She was treated with muscle relaxants and anti-inflammatory agents with little improvement. Twenty days later the painless swelling persisted and she was admitted to the hospital. Physical examination disclosed fullness and increased diameter of the left arm with cyanosis, oedema and dilation of subcutaneous collateral veins. There were no palpable lymph nodes, and the Adson manoeuvre was negative. Chest X-ray did not show abnormalities. Other investigations, including full blood count, sedimentation rate, serum biochemistry, urine analysis, coagulation study, serum electrophoresis, immunoglobulins, complement, C-reactive protein, rheumatoid factor and antinuclear antibodies, were either normal or negative. A venographic study of the left superior limb was performed (figure 1).

\author{
Hospital Juan \\ Canalejo, A Coruña, \\ Spain \\ Unidad de Corta \\ Estancia Médica \\ F de la Iglesia Martínez \\ C Pellicer Vázquez \\ V Ramos Polledo \\ F Diz-Lois Martínez \\ Servicio de \\ Radiodiagnóstico \\ M Marini Díaz \\ Servicio de \\ Hematología \\ F López Fernández \\ Correspondence to \\ $F$ de la Iglesia Martinez, \\ UCEM - $3^{a}$ planta, Hospital \\ Juan Canalejo, Xubias de \\ Arriba, 84, 15006 A \\ Coruña, Spain
}

Accepted 23 April 1998

\section{Questions}

1 What abnormality does the venographic study show and what is the diagnosis?

2 What secondary forms of this illness must be excluded?

3 What are the options for treatment? 
Answers

QUESTION 1

The venographic study confirmed the presence of axillary-subclavian vein thrombosis (figure 1). Primary forms have been called thrombosis of effort, stress thrombosis, spontaneous thrombosis and idiopathic or Paget-Schroetter syndrome, reflecting the fact that there is no unique pathogenic mechanism. ${ }^{1}$ The thrombosis of the subclavio-axillary veins comprises $1-2 \%$ of venous thrombotic events. ${ }^{2}$

\section{QUESTION 2}

Secondary forms are frequent and include venous catheterisation, drug addiction, tumour compression, intrathoracic infections, hypercoagulability states, systemic and autoimmune illnesses.

\section{QUESTION 3}

The possible treatments of primary axillary vein thrombosis are anticoagulants, local or systemic fibrinolysis, surgery, balloon angioplasty or stenting for residual stenosis, or any combination. In patients without underlying causes the commonest treatment is in fact to do nothing, other than possibly use a compression garment. This is because the majority of these patients show some spontaneous improvement, at least to a state in which the symptoms are tolerable.

\section{Follow-up}

The patient was treated with urokinase 250 $000 \mathrm{IU}$ as an intravenous bolus injection, followed by a perfusion of 100000 IU hourly. The next day another venography showed permeability. The urokinase perfusion was discontinued and heparin was given followed by oral anticoagulants. A thoracic computed tomography (CT) scan did not show mediastinal lesion or bone abnormalities.

The arm became thinner and recovered its normal colour. The dilation of the superficial veins reduced. She was put on oral anticoagulants for three months, remaining asympto-

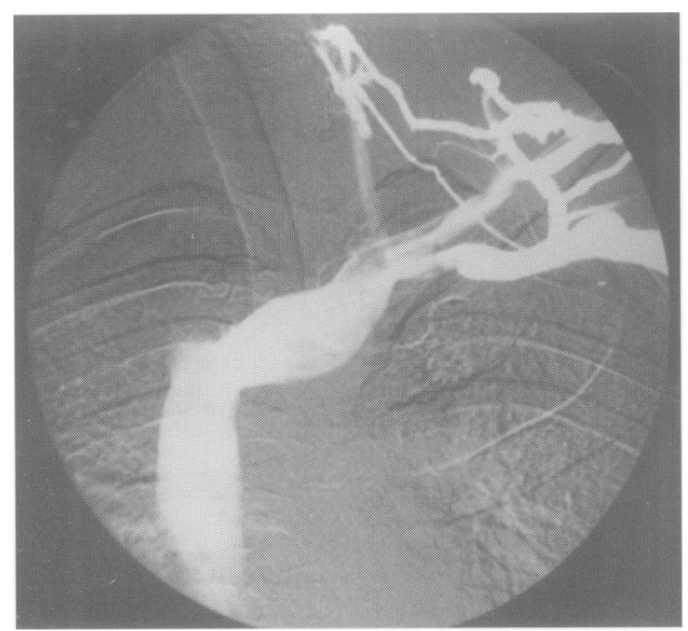

Figure 2 Residual stenosis of the subclavian vein

\section{Summary/learning points}

- most cases of axillary-subclavian vein thrombosis have a history of significant local strain or trauma; there is often underlying abnormal anatomy

- the Adson manoeuvre is the most cost-effective clinical test to explore the thoracic outlet syndrome, but it can be seen in normal individuals

- there are no unified criteria to decide which treatment is more appropriate; there is probably a place for each of them

- in selected patients, thrombolytic therapy may have an important role

matic with normal physical findings. The venogram showed a residual stenosis of the subclavian vein (figure 2). After oral anticoagulant therapy was discontinued, the patient remained asymptomatic and coagulation studies did not show evidence of hypercoagubility.

\section{Discussion}

Between $50 \%$ and $75 \%$ of cases of axillarysubclavian vein thrombosis have a history of significant local strain or trauma. ${ }^{3}$ In these patients there is often underlying abnormal anatomy. ${ }^{1}$ Our patient recalled a brisk movement of the neck before the appearance of the symptoms.

The diagnosis can be confirmed by a venogram. Other less invasive procedures, such as CT or magnetic resonance imaging, have either not been used frequently enough to assess their value or they have low sensitivity. Over the past decade, real-time B-mode ultrasonography, duplex (Doppler-directed) ultrasonography, and colour Doppler ultrasound imaging have been used more extensively as screening procedures. ${ }^{1}$ The availability of venography at the time of the evaluation of our patient, prompted us to use it as the initial investigation.

Once the diagnosis is confirmed and other secondary causes excluded, it is necessary to look for the presence of the thoracic outlet syndrome as a possible cause. Whilst the Adson manoeuvre is the most cost-effective clinical test for this purpose, a positive result is sometimes found in normal individuals. ${ }^{3}$ In our case, the clinical data and the analytical results, allowed us to exclude secondary causes and the CT scan of the thorax did not show a cervical rib or other anatomical variations.

The possible treatments of primary axillary vein thrombosis are anticoagulants, local or systemic fibrinolysis, surgery, balloon angioplasty or stenting for residual stenosis, or any combination of these. There are no unified criteria to help decide which treatment option is more appropriate since the pathogenesis is multifactorial, the reported series are short, and the follow-up is incomplete. ${ }^{134}$ Each patient must be individually assessed. ${ }^{1}$ Surgical decompression or venous by-pass are options but only appropriate for those with severe residual 
symptoms. Balloon angioplasty is effective if the underlying pathology is a embryonic web but a diffuse stenosis does not respond well. Our patient was treated medically with fibrinolytics and anticoagulants.

Pulmonary embolisation is an important complication of axillary-subclavian deep venous thrombosis, having been reported in $9.4 \%$ of patients in one study. ${ }^{4}$ In one patient it occurred immediately after thrombolytic therapy. ${ }^{5}$ In $17-75 \%$ of cases with axillary vein

1 Haire WD. Arm vein thrombosis. Clin Chest Med 1995;16:341-51.

2 De Weese JA. Management of subclavian venous obstruction. In: Beran J, Yao JS, eds. Surgery of veins. New York: Harcourt Brace Jovanovich Publishers, 1985; pp 365-83. 3 González Quijada S, Pieltain Alvarez-Arenas R, Gallego Beuter JJ, Escartin Muñoz-Ortiz P, Estrada Pérez V. Tromtres caves e individualización del tratamiento. Rev Clin Esp 1992;190:254-7. thrombosis, symptoms of chronic venous insufficiency may remain. ${ }^{34}$ In our case there were no sequelae, in spite of a residual stenosis of the vein.

\section{Final diagnosis}

Stress thrombosis of the axillary-subclavian vein

Keywords: stress thrombosis; axillary-subclavian vein; fibrinolysis

4 Becker DM, Philbrick JT, Walker FB. Axillary and subclavian venous thrombosis. Arch Intern Med 1991;151: 1934-43.

5 Jones JC, Balkcom IL, Worman RK. Pulmonary embolus after treatment for subclavian-axillary vein thrombosis. Postgrad Med F 1987;82:244-9. 